\title{
Enhanced power bacillus Calmette-Guérin-possibly too much of a good thing
}

\author{
Wojciech Krajewski ${ }^{1}$, Sławomir Poletajew ${ }^{2}$ \\ ${ }^{1}$ Department of Urology and Oncological Urology, Wrocław Medical University, Wrocław, Poland; ${ }^{2}$ Department of General, Oncological and \\ Functional Urology, Medical University of Warsaw, Warsaw, Poland \\ Correspondence to: Wojciech Krajewski. Department of Urology and Oncological Urology, Wroclaw Medical University, Borowska 213, Wroclaw, \\ Poland. Email: wk@softstar.pl. \\ Provenance: This is an invited article commissioned by the Section Editor Xiao Li (Department of Urology, Jiangsu Cancer Hospital \& Jiangsu \\ Institute of Cancer Research \& Nanjing Medical University Affiliated Cancer Hospital, Nanjing, China). \\ Comment on: Rodriguez D, Goulart C, Pagliarone AC, et al. In vitro Evidence of Human Immune Responsiveness Shows the Improved Potential of a \\ Recombinant BCG Strain for Bladder Cancer Treatment. Front Immunol 2019;10:1460.
}

Submitted Sep 23, 2019. Accepted for publication Oct 06, 2019.

doi: $10.21037 /$ tau. 2019.10 .08

View this article at: http://dx.doi.org/10.21037/tau.2019.10.08

Intravesical bacillus Calmette-Guérin (BCG) immunotherapy is the standard adjuvant treatment in patients with intermediate- and high-risk non-muscle invasive bladder cancer (NMIBC) after transurethral resection of the bladder tumour (TURB). It was widely proven, that this therapy reduces the risk of tumour recurrence and, what is more important, the risk of cancer progression. However, despite decades of BCG usage, precise mechanism, optimal schedule, dosage and various other details remain unknown. Furthermore, some patients do not respond well to the therapy, while others develop side effects. Finally, we have to face the BCG shortage because of production and logistics issues (1). All these factors motivate and promote the search for BCG alternatives or classic BCG ameliorations.

In the paper by Rodriguez et al. published recently in the Frontiers in Immunology Journal authors analyse biological and immunological potential of recombinant BCG Moreau strain (2). Authors should be praised for preparing methodologically sound study assessing the influence of bacilli on many different areas of immunological response. The provided data proves that $\mathrm{rBCG}-\mathrm{S} 1 \mathrm{PT}$ strain enhances immune activation when compared to wild type BCG in terms of cytokine production and tumour cytotoxicity.

Yet, there is the other side of the coin. It should be emphasized that alike BCG effectiveness, BCG toxicity is generated by immunological properties of bacilli. Those two phenomena are somehow link and therefore, some authors advocate the "no pain-no gain" hypothesis which state that the higher the toxicity the better are the oncological results. However, the pathogenesis of adverse reactions following intravesical BCG instillation has not been fully explained. Having all that in mind, one should assume, that enhancing the immune-inducing properties of the intravesical instillation will probably increase the treatment toxicity. As we know from cohort studies, almost all patients experience some side effects, whereas they are graded as severe in as many as $30 \%$ of cases. Because of that, in many cases, side effects of BCG therapy impose dose reduction or force treatment cessation (3). Therefore, it is possible, that additional BCG strengthening will significantly limit its application options.

As authors highlight in their paper, the in vitro immunological response to the intravesical BCG therapy varies significantly between individuals. This is also obvious in clinical setting. We all know that despite implementation of "proper" BCG schedule, in some patients the oncological response is not optimal and the disease progresses. This raises the necessity to find the definitive causes of variety phenomenon, or at least precise predictive factors of therapy outcome. Unfortunately, despite intensive search, all analysed factors have proved limited efficacy (at best). From studies on muscle invasive bladder cancer, it seems, that the most promising future prediction models will be based on genomic profile and genomic biomarkers, e.g., 
mutations in DNA damage and repair genes (4). As it was recently shown, that about $30 \%$ of high-grade NMIBCs harbour these mutations (5).

The next interesting issue raised by the authors concerns influence of previous BCG immunisation. In the available literature we can find various papers analysing this problem, yet, the result is conflicting and the ultimate answer is unknown. Authors of this study show that there were differences in cytokines levels between vaccinated and nonvaccinated patients. Yet, the conclusion should be drawn with caution as the examined population was very small and as vaccination is not the sole origin of mycobacterial immunization.

Finally, recombination of BCG bacilli does not respond to the problem of BCG shortage, which nowadays seems to be the most significant BCG-related issue in Europe. Researchers focus more and more on establishing alternative regimens instead of improving BCG. Even the most effective BCG recombination would remain useless if BCG would not be available.

Last but not least, the in vitro results do not always translate directly into clinics, so we need to cross our fingers for upcoming clinical trial on rBCG-S1PT and hold our optimism until we see their outcomes.

\section{Acknowledgments}

None.

Cite this article as: Krajewski W, Poletajew S. Enhanced power bacillus Calmette-Guérin-possibly too much of a good thing. Transl Androl Urol 2019;8(Suppl 5):S461-S462. doi: 10.21037/tau.2019.10.08

\section{Footnote}

Conflicts of Interest: The authors have no conflicts of interest to declare.

Ethical Statement: The authors are accountable for all aspects of the work in ensuring that questions related to the accuracy or integrity of any part of the work are appropriately investigated and resolved.

\section{References}

1. Messing EM. The BCG Shortage. Bladder Cancer 2017;3:227-8

2. Rodriguez D, Goulart C, Pagliarone AC, et al. In vitro Evidence of Human Immune Responsiveness Shows the Improved Potential of a Recombinant BCG Strain for Bladder Cancer Treatment. Front Immunol 2019;10:1460.

3. Liu Y, Lu J, Huang Y, et al. Clinical Spectrum of Complications Induced by Intravesical Immunotherapy of Bacillus Calmette-Guerin for Bladder Cancer. J Oncol 2019;2019:6230409.

4. Hedegaard J, Lamy P, Nordentoft I, et al. Comprehensive Transcriptional Analysis of Early-Stage Urothelial Carcinoma. Cancer Cell 2016;30:27-42.

5. Liu D, Plimack ER, Hoffman-Censits J, et al. Clinical Validation of Chemotherapy Response Biomarker ERCC2 in Muscle-Invasive Urothelial Bladder Carcinoma. JAMA Oncol 2016;2:1094-6. 